\title{
ON THE DENSITY OF THE CUT POINTS AND END POINTS OF A CONTINUUM*
}

\author{
BY W. L. AYRES
}

1. Introduction. We consider a connected, compact, metric space $M$ which we refer to as the continuum $M$. The space $M$ is said to be locally connected if for each point $p$ of $M$ and each $\epsilon>0$ there exists a $\delta>0$ and a connected set $N$ such that $S(p, \delta) \subset N \subset S(p, \epsilon)$. A point $p$ is said to be a cut point of $M$ if $M-p$ is not connected. A point $p$ is said to be an end point of $M$ if for each $\epsilon>0$ there exists a neighborhood $U_{p}$ such that $U_{p} \subset S(p, \epsilon)$ and $B\left(U_{p}\right)=\bar{U}_{p}-U_{p}$ is a single point. $\dagger$ From this definition it is seen that every end point is a limit point of the cut points of $M$. Hence whenever the end points are dense in $M$, the cut points are also dense. This relation is not true conversely, but a study of some examples leads one to the conclusion that there exist some fundamental relations between the density of the cut points and the density of the end points. In this note we shall investigate some of these relations.

2. Notation. Let $K$ and $E$ denote respectively the set of all cut points and end points of $M$. Let $K^{2}$ denote the set of all cut points which are of Urysohn-Menger order 2 in $M$ and let ${ }^{2} \mathrm{~K}$ denote the set of all cut points which are of order $>2$. Capitals will denote sets of points, lower case letters single points. $S(p, \epsilon)$ denotes the set of all points whose distance from $p$ is less than $\epsilon$. The symbol $\rho(x, y)$ denotes the distance from $x$ to $y ; \rho(X, Y)$ denotes the greatest lower bound of the numbers $\rho(x, y)$ where $x \varepsilon X$ and $x \varepsilon Y$. The notations $x \varepsilon X$ and $x$ non- $\varepsilon X$ mean " $x$ is a point of the set $X$ " and " $x$ is not a point of $X$ " respectively. The symbol $d(X)$ denotes the diameter of $X$, that is, the least upper bound of all numbers $\rho(x, y)$ where $x \varepsilon X$ and $y \varepsilon X$.

\footnotetext{
* Presented to the Society, August 30, 1929.

$\dagger$ We use end point here in the Urysohn-Menger sense. See P. Urysohn, Comptes Rendus, vol. 175 (1922), pp. 481-48,3; and K. Menger, Mathematische Annalen, vol. 95 (1925), pp. 277-306. For other senses in which the term has been used, see H. M. Gehman, Concerning end points of continuous curves and other continua, Transactions of this Society, vol. 30 (1928), pp. 63-84.
} 
3. ThEOREM. If the end points are dense in $M-K$, the cut points are dense in $M$.

Since each end point is a limit point of the cut points, we have $\bar{E} \subset \bar{K}$. By hypothesis, $M-K \subset \bar{E}$. Then $M-K \subset \bar{K}$. As $K \subset \bar{K}$, we have $M=\bar{K}$.

4. THEOREM. In order that the cut points of a locally connected continuum $M$ be dense in $M$ it is necessary and sufficient that the end points be dense in $M-K . \quad[(\bar{K}=M) \equiv(\bar{E} \supset M-K)$.

The condition is sufficient by the preceding theorem. Now let $p \varepsilon M-K-E$ and choose $\epsilon>0$. There exists just one maximal cyclic set $C$ of $M$ containing $p$. $^{*}$ By hypothesis there exists a $q_{1}^{\prime} \varepsilon K \cdot S(p, \epsilon / 2)$. If $q_{1}^{\prime} \varepsilon C$, let $q_{1}=q_{1}^{\prime}$ and let $H_{1}$ be a component of $M-q_{1}$ such that $H_{1} \cdot C=0$. If $q_{1}^{\prime}$ non- $\varepsilon C$, let $H_{1}$ be the component of $M-C$ containing $q_{1}^{\prime}$ and let $q_{1} \varepsilon C \cdot \bar{H}_{1}$. Evidently $q_{1}=C \cdot \bar{H}_{1}$ and $q_{1} \neq p$. There exists an integer $m_{2}>2$ such that $S\left(p, \epsilon / m_{2}\right) \cdot H_{1}=0$. Let $q_{2}^{\prime} \varepsilon K \cdot S\left(p, \epsilon / m_{2}\right)$. If $q_{2}^{\prime} \varepsilon C$, let $q_{2}=q_{2}^{\prime}$ and $H_{2}$ be a component of $M-q_{2}$ such that $H_{2} \cdot C=0$. If $q_{2}^{\prime}$ non- $\varepsilon C$, let $H_{2}$ be the component of $M-C$ containing $q_{2}^{\prime}$ and let $q_{2}=C \cdot \bar{H}_{2}$. We see quite easily that $H_{2} \cdot H_{1}=0$ and $q_{2} \neq p$. In general there exists an integer $m_{i}>m_{i-1}(i>1)$ such that $S\left(p, \epsilon / m_{i}\right) \cdot H_{i-1}=0$. Let $q_{i}^{\prime} \varepsilon K \cdot S\left(p, \epsilon / m_{i}\right)$ and we find $H_{i}$ and $q_{i}$ from this exactly as above. Again we have $q_{i} \neq p$ and $H_{i} \cdot H_{j}=0$. Then we have an infinite set of distinct components of $M-C, H_{1}, H_{2}, H_{3}, \cdots$, such that

$$
\begin{gathered}
\bar{H}_{i} \supset q_{i}^{\prime}, \quad \lim _{i \rightarrow \infty} q_{i}^{\prime}=p, \\
\bar{H}_{i} \cdot C=q_{i} \neq p .
\end{gathered}
$$

Since $M$ is locally connected it follows from (1) and (2) that

$$
\lim _{i \rightarrow \infty} q_{i}=p .
$$

Now as $d\left(H_{i}\right)$ approaches zero it follows from (3) that there

* We are using the term maximal cyclic set in place of Whyburn's term maximal cyclic curve. For definitions and properties of maximal cyclic sets, see G. T. Whyburn, Cyclicly connected continuous curves, Proceedings of the National Academy of Sciences, vol. 13 (1927), pp. 31-38. These results are extended to metric space in my paper, Concerning continuous curves in metric space, American Journal of Mathematics. vol. 51 (1929), pp. 577-594. 
exists an integer $n$ such that $S(p, \epsilon) \supset H_{n}$. If $\bar{H}_{n}$ is cyclicly connected, then $\bar{H}_{n} \cdot K=q_{n}$. Then no point of $H_{n}$ is a limit point of $K$ contrary to hypothesis. Then $\bar{H}_{n}$ is not cyclicly connected and thus contains two nodes of itself.* One of these nodes $N$ does not contain $q_{n}$. Either $N$ is an end point of $\bar{H}_{n}$ or $N$ is a maximal cyclic set of $\bar{H}_{n}$ containing just one cut point of $\bar{H}_{n}$. But in this last case $N$ is a maximal cyclic set of $M$ and contains just one point of $K$. But this is impossible just as above. Hence $N$ is an end point of $\bar{H}_{n}$ and it is not difficult to see that $N \varepsilon E$. Then for each $\epsilon>0$ there exists an $N \varepsilon E \cdot S(p, \epsilon)$. Hence $p \varepsilon \bar{E}$.

5. Example. We may see quite simply that the condition of the preceding theorem is not necessary without the restriction that $M$ be locally connected. Let $M$ be the curve $y=\sin 1 / x$ for $0<x \leqq 1$ together with the points of the $y$-axis for which $-1 \leqq y \leqq 1$. Then $\bar{K}=M$ but the points of $M$ on the $y$-axis belong to $M-K$ and no one of them belongs to $\bar{E}$. In fact $E$ is the single point $(1, \sin 1)$.

6. THEOREM. In order that the end points of a locally connected continuum $M$ be dense in $M$ it is necessary and sufficient that the cut points of order $>2$ be dense in $M . \quad\left[(\bar{E}=M) \equiv\left({ }^{2} \bar{K}=M\right).\right]$

The condition is necessary. Since $E \neq 0$, the continuum $M$ is not cyclicly connected. Then if $M$ contains a maximal cyclic set $C$, each component of $M-C$ has just one limit point in $C$ and thus $C \cdot K \neq 0$. Further $C \cdot K=C \cdot{ }^{2} K$, for if $x \varepsilon C \cdot K$ there exist at least three arcs meeting at $x$ (two arcs on a simple closed curve of $C$ containing $x$ and one arc in a component of $M-C$ whose limit point is $x$ ) and so $x$ is of order $\geqq 3$ in $M$.

Now let $p \varepsilon E$. If $p$ non- $\varepsilon^{2} \bar{K}$, there exists a neighborhood $U_{p}$ such that $U_{p} \cdot{ }^{2} K=0$. As $p \varepsilon E$ there exists a neighborhood $V_{p}$ such that $\bar{V}_{p} \subset U_{p}$ and $B\left(V_{p}\right)=q$, a single point. Let $A_{1}$ be an arc of $M$ with end points $p$ and $q$ and let $y \varepsilon A_{1}-p-q$. Evidently $A_{1} \subset \bar{V}_{p}$. From the local connectivity there exists a neighborhood $W_{y} \subset V_{p}-p$ such that if $z \varepsilon W_{y}$ then there is an arc from $z$ to $y$ which lies in $V_{p}$. As $\bar{E}=M$ let $z \varepsilon E \cdot W_{y}$. Then $z \varepsilon M-A_{1}$. Let $A_{2}$ be the arc of $M$ joining $z$ and $y$ such

* G. T. Whyburn, Concerning the structure of a continuous curve, American Journal of Mathematics, vol. 50 (1928), pp. 167-194, Theorem 14. 
that $V_{p} \supset A_{2}$. In the order from $z$ to $y$ let $w$ be the first point of $A_{1}$ on $A_{2}$. The point $w \neq p$ for $p \varepsilon E$ and thus is not interior to an arc of $M$. The point $w$ is of order $\geqq 3$ since there are at least three arcs (subarc wp of $A_{1}$, subarc $w q$ of $A_{1}$, subarc $w z$ of $A_{2}$ ) meeting in $w$. Then $w$ non- $\varepsilon K$ since $V_{p} \cdot{ }^{2} K=0$. As $w$ non- $\varepsilon K$ there exists an arc $A_{3} \subset M-w$ with end points $z$ and $p$. It is easy to see that $\bar{V}_{p} \supset A_{3}$. From the set $A_{1}+A_{2}+A_{3}$ we may obtain a simple closed curve $J \subset \bar{V}_{p}$. Let $C$ be the maximal cyclic set containing $J$. In the preceding paragraph we saw that $C \cdot{ }^{2} K \neq 0$. But $C \subset \bar{V}_{p} \subset U_{p}$ and $U_{p} \cdot{ }^{2} K=0$. Then the assumption that $p$ non- $\varepsilon{ }^{2} \bar{K}$ leads to an absurdity. Hence $E \subset{ }^{2} \bar{K}$. But $M=\bar{E}$. Then $M={ }^{2} \bar{K}$, which is the desired relation.

The condition is sufficient. From the result of $\$ 4$ we have the set $F=M-K \subset \bar{E}$ and thus $\bar{F} \subset \bar{E}$. If $C$ is a maximal cyclic set of $M$, all but a countable number of points of $C$ belong to $M-K$. Then $C \subset \bar{F}$. Let $p \varepsilon M-\bar{F}$ which is of order 2 in $M$ and let $U_{p}$ be a neighborhood of $p$. There exists a number $\epsilon_{1}>0$ such that $S\left(p, \epsilon_{1}\right) \cdot \bar{F}=0$ and $S\left(p, \epsilon_{1}\right) \subset U_{p}$. If $J$ is any simple closed curve of $M, S\left(p, \epsilon_{1}\right) \cdot J=0$. As $p \varepsilon K$ there is an $\operatorname{arc} A \subset U_{p}$ and containing $p$ as an interior point. Since ${ }^{2} \bar{K}=M$, there is a point $q_{1} \varepsilon^{2} K \cdot S\left(p, \boldsymbol{\epsilon}_{1}\right)$. If $q_{1} \varepsilon A$, let $x_{1}=q_{1}$. In this case there is a component $H_{1}$ of $M-A$ such that $\bar{H}_{1} \cdot A=q_{1}=x_{1}$. If $q_{1}$ non- $\varepsilon A$, let $H_{1}$ be the component of $M-A$ containing $q_{1}$. The set $\bar{H}_{1} \cdot A$ is a single point $x_{1}$ for otherwise there exists a simple closed curve $J$ such that $J \cdot S\left(p, \epsilon_{1}\right) \neq 0$. Since $p$ is of order $2, p \neq x_{1}$. There exists a number $0<\epsilon_{2}<\frac{1}{2} \epsilon_{1}$ such that $S\left(p, \epsilon_{2}\right) \cdot \bar{H}_{1}=0$. Let $q_{2} \epsilon^{2} K \cdot S\left(p, \epsilon_{2}\right)$. If $q_{2} \varepsilon A$, let $q_{2}=x_{2}$ and there is a component $H_{2}$ of $M-A$ such that $\bar{H}_{2} \cdot A=q_{2}=x_{2}$. If $q_{2}$ non- $\varepsilon A$, let $H_{2}$ be the component of $M-A$ containing $q_{2}$ and let $x_{2} \varepsilon \bar{H}_{2} \cdot A$. As above $x_{2}=\bar{H}_{2} \cdot A$ since $J \cdot S\left(p, \epsilon_{1}\right)=0$ for every simple closed curve $J$. Continue this process indefinitely. In general there exists a number $0<\epsilon_{n}<\frac{1}{2} \epsilon_{n-1}$ such that $S\left(p, \boldsymbol{\epsilon}_{n}\right) \cdot\left(\bar{H}_{1}+\bar{H}_{2}+\cdots+\bar{H}_{n-1}\right)=0$. Let $q_{n} \varepsilon^{2} K \cdot S\left(p, \boldsymbol{\epsilon}_{n}\right)$ and define $x_{n}$ and $H_{n}$ as above. Then $x_{n} \neq p$.

From the connectedness im kleinen of $M$ we have

$$
\lim _{n \rightarrow \infty} x_{n}=p, \quad \lim _{n \rightarrow \infty} d\left(H_{n}\right)=0 .
$$

From this it follows that there exists an integer $m$ such that $H_{m} \subset S\left(p, \epsilon_{1}\right)$. The locally connected continuum $\bar{H}_{m}$ is acyclic 
since $J \cdot S\left(p, \epsilon_{1}\right)=0$ for each simple closed curve of $M$. Then every node of $\bar{H}_{m}$ is an end point of $\bar{H}_{m}$. This set contains at least two nodes and thus there is a point $z \neq x_{m}$ which is an end point of $\bar{H}_{m}$. It is clear that $z \varepsilon E$. Then $z \subset \bar{H}_{m} \subset S\left(p, \epsilon_{1}\right) \subset U_{p}$. Therefore $p \epsilon \bar{E}$.

We have $M-K+K^{2} \subset \bar{E}$, and $M-\left(M-K+K^{2}\right)={ }^{2} K$. By a theorem of G. T. Whyburn, ${ }^{*}$ the set ${ }^{2} K$ is countable and thus contains no open subset of $M$. For this reason ${ }^{2} K \subset \bar{E}$.

7. The Necessity of Local Connectivity. We shall show now that the condition of the preceding theorem is neither necessary nor sufficient unless $M$ is locally connected. That the condition is not sufficient follows from the first two properties of a continuum $M$ described in the next paragraph.

There exists a continuum $M$ with the following properties: (1) $M$ has no end point, (2) the cut points of order $>2$ are dense in $M$, (3) the set of cut points of order 2 is an uncountable zerodimensional set which is dense in $M$.

Let $a, b, c$ be the points $(0,0),(1,1),(1,-1)$. Let $M_{1}=a b c$, where by such a symbol we will mean the set of all points of the triangle plus all interior points. Let $x_{i}$ be the number $1-1 / 2^{i}$. Let $y_{i}$ be the $i$ th number in the series $0, \frac{1}{2}, 0,-7 / 8, \cdots$, that is, $y_{i}=0, x_{i-1}$ or $-x_{i-1}$ according as $i$ is of the form $2 n+1$, $4 n+2$ or $4 n$. Let $p_{i}(i=1,2, \cdots)$ be the point $\left(x_{i}, y_{i}\right)$. Let $u_{i}$ and $v_{i}(i=0,1,2, \cdots)$ be the upper and lower end points of a vertical segment of length $1 / 2^{i}$ with $p_{i+1}$ as mid-point. Let $p_{0}=a$. Let

$$
M_{2}=b c+\sum_{i=0}^{\infty} p_{i} u_{i} v_{i}
$$

Now let $T_{i}$ be the collineation such that $T_{i}(a)=p_{i}, T_{\imath}(b)=u_{i}$, $T_{i}(c)=v_{i}$. Let

$$
M_{3}=\sum_{i=0}^{\infty} T_{i}\left(M_{2}\right) .
$$

In general let $\Delta$ be a maximal triangle of $M_{n-1}$ and $p_{\Delta}, u_{\Delta}$ and $v_{\Delta}$ be the left-, upper right-, and lower right-hand vertices of $\Delta$. Let $T_{\Delta}$ be the collineation such that $T_{\Delta}(a)=p_{\Delta}, T_{\Delta}(b)=u_{\Delta}$, $T_{\Delta}(c)=v_{\Delta}$, and let

* G. T. Whyburn, Concerning collections of cuttings of connected point sets. this Bulletin, vol. 35 (1929), pp. 87-104, Theorem 13. 


$$
M_{n}=\sum T_{\Delta}\left(M_{2}\right)
$$

for every $\Delta \subset M_{n-1}$. Now let

$$
G=\prod_{n=1}^{\infty} \bar{M}_{n} .
$$

The set $G$ is a continuum such that every vertical line $x=k(0 \leqq k \leqq 1)$ intersects it in a single point or in an interval which is a side of one of the triangles $\Delta$ at some stage. The mid-point of each of these intervals (except $b c$ ) is a cut point of $G$ of infinite order due to the oscillation of the set to the left of the point. It is easily seen that these cut points are dense in $G$. The point $a$ is the only end point of $G$. Let $H$ be the reflection of $G$ in the $y$-axis, and let $M=G+H$. Then the continuum $M$ has properties (1) and (2).

Except for a countable number of values of $k$, the line $x=k(-1 \leqq k \leqq 1)$ intersects $M$ in a single point. Let $q$ be any such point. The point $q=a$ is obviously a cut point of order 2 as it is of order 1 in both $G$ and $H$. If $q \neq a$, then $q$ is in terior to some such point. Then $q=a$ is obviously a cut point of order 2 as it is of order 1 in both $G$ and $H$. If $q \neq a$, then $q$ is interior to some triangle $\Delta$ of $M_{n}$ at each stage. If $\epsilon>0$, there exists an integer $n$ such that the triangle $\Delta$ of $M_{n}$ containing $q$ is of diameter $<\frac{1}{2} \epsilon$ and $a$ non- $\varepsilon \Delta$. The triangle $\Delta$ contains just two limit points of that part of $M$ outside $\Delta$ (its left-hand vertex and the mid-point of the vertical side). From this it is easy to see that we may obtain a quadrilateral $Q$ of diameter $<\epsilon$ which has just these two points in common with $\Delta$, encloses the rest of $\Delta$, and has in its exterior $M-\Delta$. Then the interior of $Q$ is a neighborhood of $q$ of diameter $<\epsilon$ whose boundary has just two points in common with $M$. Hence $q$ is of order 2. That $\operatorname{dim}$ $K^{2}=0$ follows at once for both points of $M \cdot B(Q)$ belong to ${ }^{2} K$. This gives $\operatorname{dim}_{q} K^{2}=0$ for $q \neq a$. It is also easy to see that $\operatorname{dim}_{a} K^{2}=0$.

We shall proceed to show now that the condition of the preceding theorem is not necessary unless $M$ is locally connected, that is,

There exists a continuum $M$, which contains no cut point of order $>2$, and yet the end points are dense in $M$.

Let $\left[r_{n}\right]$ denote the rational points in the interval $(0,1)$, 
except 0 , arranged in a sequence. Let $f_{n}(x)=\left(1 / n^{2}\right)\left[\sin 1 /\left(x-r_{n}\right)\right]$ for $x \neq r_{n} ; f_{n}(x)=0$ for $x=r_{n}(0 \leqq x \leqq 1)$. Let

$$
F(x)=\sum_{n=1}^{\infty} f_{n}(x) .
$$

Let $H$ be the set composed of the graph of $F(x)$ and its limit points. ${ }^{*}$ The following properties of $I I$ may be easily verified: (a) $H$ is a continuum, (b) every line $x=k(0 \leqq k \leqq 1)$ intersects $I I$ in either a point or an interval, (c) every such point is a cut point of $H$ of order 2, (d) the set of all such intervals is a countable set, (e) $H$ contains no cut point of order $>2$, (f) the point (0, $F(0))$ is the single end point of $H$.

Now we may transform the set $H$ into a set $M_{1}$ such that (1) the point $(0, F(0))$ of $H$ becomes the point $a=(0,0)$, (2) the upper and lower end points of the interval of $H$ containing $(1, F(1))$ become the points $b=(1,1)$ and $c=(1,-1),(3)$ every point of $M_{1}$ lies in or on the triangle $a b c$, (4) the properties (a)-(f) remain true for $M_{1}$. In fact we may make this transformation so as not to change the $x$-coordinate of any point.

For each interval $I_{n}$ of $M_{1}$ let $W_{n}$ be a wedge-shaped region bounded by two planes through $I_{n}$ making equal angles with the $x y$-plane, two planes through the end points of $I_{n}$ perpendicular to $I_{n}$ and a plane parallel to the $x y$-plane. Evidently the regions $W_{n}$ may be taken so that

$$
\bar{W}_{i} \cdot \bar{W}_{j}=0(i \neq j) \text {, and } d\left(W_{n}\right)<1 / n .
$$

Let $\left(a_{n}, b_{n}, 0\right)$ and $\left(a_{n}, c_{n}, 0\right)$ be the end points of $I_{n}\left(b_{n}>c_{n}\right)$, and let $d_{n}$ be a number such that the point $\left(a_{n}, \frac{1}{2}\left(b_{n}+c_{n}\right), d_{n}\right)$ lies in the interior of $W_{n}$. Let $\Psi_{n}$ be the transformation $x^{\prime}=a_{n}$, $y^{\prime}=\frac{1}{2}\left(b_{n}+c_{n}\right)+\frac{1}{2}\left(b_{n}-c_{n}\right) y, z^{\prime}=-d_{n} x+d_{n}$. Let

$$
M_{2}=\sum_{n=1}^{\infty} \Psi_{n}\left(M_{1}\right) \text {. }
$$

* See W. A. Wilson, On the structure of a continuum, limited and irreducible between two points, American Journal of Mathematics, vol. 48 (1926), p. 162. The continuum $H$ may also be constructed by methods similar to the previous example by building up oscillations both right and left. However the description becomes rather intricate. Also the previous example may be set up by the Cantor method of condensation of singularities but its properties appear somewhat clearer by the use of the triangles $\Delta$. 
Let $I_{n m}$ be the intervals of $\Psi_{n}\left(M_{1}\right)$ except for $I_{n}$, and for each $I_{n m}$ we take a wedge-shaped region $W_{n m}$ such that $\bar{W}_{n i} \cdot \bar{W}_{n j}$ $=0(i \neq j), d\left(W_{n m}\right)<1 /(n+m), W_{n m} \cdot\left(M_{1}+M_{2}\right)=I_{n m}, \bar{W}_{n m} \subset$ interior $W_{n}$. Now we define a linear transformation $\Psi_{n m}$ such that $\Psi_{n m}\left(M_{1}\right)$ is a set homeomorphic with $M_{1}$ and lying in $W_{n m}$ except for $I_{n m}$, just as above. Let

$$
M_{3}=\sum_{n} \sum_{m} \Psi_{n m}\left(M_{1}\right) .
$$

Continue this process indefinitely. Now let

$$
M=\sum_{i=1}^{\infty} M_{i} .
$$

The set $\bar{M}$ is the required example. To simplify the description as far as possible we have defined $\bar{M}$ as lying in euclidean three-space. However, on careful examination of $\bar{M}$ it may be seen that $\bar{M}$ can be mapped in the plane.

8. Theorem. If the end points are dense in $M$, then $\operatorname{dim} M^{2} \leqq 0$.

In a paper not yet published, ${ }^{*}$ I have proved that for the set $M^{2}$, that is, the points of $M$ of order 2, we have the following relation:

$$
M^{2}=H+G,
$$

where (1) $\operatorname{dim} H \leqq 0,(2) G$ is a vacuous or countable set of $\operatorname{arcs} A_{i}$, (3) each arc-segment $A_{i}$ is an open subset of $M$. Now if we suppose that $\operatorname{dim} M^{2}>0$, it follows from this result that the set $G$ contains at least one arc $A_{1}$. Let $p$ be an interior point of $A_{1}$. From property (3) there exists a neighborhood $U_{p} \subset A_{1} \subset M^{2}$. Hence $U_{p} \cdot E=0$ contrary to the hypothesis of our theorem.

Corollary. If the end points are dense in $M$, then $\operatorname{dim} K^{2} \leqq 0$.

9. ThEOREM. In order that the end points of a locally connected continuum $M$ be dense in $M$ it is necessary and sufficient that the cut points be dense in $M$ and $\operatorname{dim} K^{2} \leqq 0 . \quad[(\bar{E}=M) \equiv(\bar{K}=M)$ $\cdot\left(\operatorname{dim} K^{2} \leqq 0\right)$.]

* On the regular points of a continuum. This paper has been submitted to the Transactions of this Society. See an abstract in this Bulletin, vol. 36 (1930), p. 485. 
The conditions of the theorem are necessary by the results of $\S 3$ and the corollary of $\S 8$. We may show that the conditions are sufficient by an argument following closely that used in proving the sufficiency of the condition in $\$ 6$.

The first example of $\$ 7$ shows that the conditions of the theorem are not sufficient if we do not specify that $M$ is locally connected.

The University of Michigan

\section{ON THE GENERALIZATION OF TRIGONOMETRIC IDENTITIES IN ARITHMETICAL PARAPHRASING* \\ BY H. T. ENGSTROM $\dagger$}

1. Introduction. Identities of the type

$$
\sum_{s=1}^{m} \alpha_{s} \sin a_{s} x \equiv \sum_{t=1}^{n} \beta_{t} \sin b_{t} x
$$

where $\alpha_{s}, a_{s}, \beta_{t}, b_{t}$ are rational integers, arise in the comparison of like powers of the modulus when an elliptic function is represented in more than one way by trigonometric series. The following theorem is used in obtaining arithmetical results from such identities.

THEOREM 1. If $g(x)$ is an arbitrary, single-valued, odd function, defined for $x=a_{s}, s=1,2, \cdots, m$, and $x=b_{t}, t=1,2, \cdots, n$, then (1) implies

$$
\sum_{s=1}^{m} \alpha_{s} g\left(a_{s}\right)=\sum_{t=1}^{n} \beta_{t} g\left(b_{t}\right)
$$

Similarly, for cosines, we have the following statement.

THEOREM 2. If $f(x)$ is an arbitrary, single-valued, even function, defined for $x=a_{s}, s=1,2, \cdots, m$, and $x=b_{t}, t=1,2, \cdots$, $n$, then

$$
\sum_{s=1}^{m} \alpha_{s} \cos a_{s} x \equiv \sum_{t=1}^{n} \beta_{t} \cos b_{t} x
$$

* Presented to the Society, April 5, 1930.

$\dagger$ National Research Fellow, California Institute of Technology. 